\title{
Mirror links have dual odd and generalized Khovanov homology
}

\author{
KRZYSZTOF K PUTYRA \\ WOJCIECH LUBAWSKI
}

\begin{abstract}
We show that the generalized Khovanov homology defined by the second author in the framework of chronological cobordisms admits a grading by the group $\mathbb{Z} \times \mathbb{Z}_{2}$, in which all homogeneous summands are isomorphic to the unified Khovanov homology defined over the ring $\mathbb{Z}_{\pi}:=\mathbb{Z}[\pi] /\left(\pi^{2}-1\right)$. (Here, setting $\pi$ to \pm 1 results either in even or odd Khovanov homology.) The generalized homology has $\mathbb{k}:=\mathbb{Z}\left[X, Y, Z^{ \pm 1}\right] /\left(X^{2}=Y^{2}=1\right)$ as coefficients, and the above implies that most automorphisms of $\mathbb{k}$ fix the isomorphism class of the generalized homology regarded as a $\mathbb{k}$-module, so that the even and odd Khovanov homology are the only two specializations of the invariant. In particular, switching $X$ with $Y$ induces a derived isomorphism between the generalized Khovanov homology of a link $L$ with its dual version, ie the homology of the mirror image $L^{!}$, and we compute an explicit formula for this map. When specialized to integers it descends to a duality isomorphism for odd Khovanov homology, which was conjectured by A Shumakovitch.
\end{abstract}

$55 \mathrm{~N} 35,57 \mathrm{M} 27$

\section{Introduction}

In his seminal paper [2], Khovanov constructed for every link $L$ a sequence of graded abelian groups $\mathcal{H}_{e v}^{i}(L)$ called the Khovanov homology of the link $L$. The graded Euler characteristic of $\mathcal{H}_{e v}(L)$ is the famous Jones polynomial $J_{L}(q)$, of which many properties have an interpretation at the higher level of homology groups. For instance, for a mirror link $L^{!}$we have $J_{L^{!}}(q)=J_{L}\left(q^{-1}\right)$, which corresponds to duality of Khovanov homology in the derived sense, ie there is an isomorphism $C\left(L^{!}\right) \stackrel{\cong}{\longrightarrow} C(L)^{*}:=\operatorname{Hom}(C(L) ; \mathbb{Z})$ between complexes of free groups that compute the homology. ${ }^{1}$ Such an isomorphism has been already explicitly constructed in [2], but its existence can be also deduced from an extension of the homology to link cobordisms constructed by Khovanov [3] and Bar-Natan [1].

\footnotetext{
${ }^{1}$ One can regard this as an isomorphism between the Khovanov cohomology of a link $L$ and the Khovanov homology of the mirror link $L^{!}$.
} 
The Khovanov homology is not the only categorification of the Jones polynomial. A distinct homology theory $\mathcal{H}_{\text {odd }}(L)$ was discovered by Ozsváth, Rasmussen and Szabó [4], which they called the odd Khovanov homology. For this reason we shall refer to the original construction as even. Both theories agree when regarded with coefficients in the two-element field $\mathbb{F}_{2}$, but they are totally different over integers: there are pairs of knots with the same homology of one type but with differing homology of the other type, see Shumakovitch [7]. Computer-based calculation revealed the duality phenomenon for the odd homology, but a theoretical explanation was missing: neither an extension to link cobordisms of the odd theory nor an explicit isomorphism between complexes was known.

Both theories were unified by one of the authors [5; 6]. The generalized Khovanov homology $\mathcal{H}(L)$ is a sequence of modules over the ring

$$
\mathbb{k}:=\mathbb{Z}\left[X, Y, Z^{ \pm 1}\right] /\left(X^{2}=Y^{2}=1\right),
$$

and both even and odd homology can be recovered by specifying integers for the generators $X, Y$ and $Z$. More precisely, the even and odd homology are isomorphic to $\mathcal{H}(L ; \mathbb{Z})$ for various $\mathbb{k}$-module structures on $\mathbb{Z}$ :

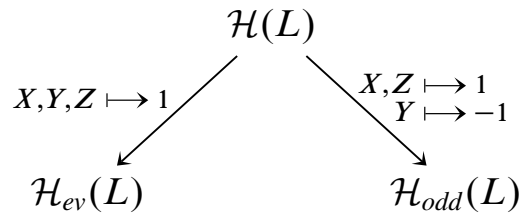

This leads into eight possible homology theories, half of which were easily shown to be redundant: multiplying the action on $\mathbb{Z}$ of each generator $X, Y$ and $Z$ by -1 does not change $\mathcal{H}(L ; \mathbb{Z})[5]$. However, four choices are left and we prove in this paper that they can be further reduced to just two cases: the even and odd homology. In particular, the most general theory in our framework, $\mathcal{H}_{\pi}(L)$, is defined over the ring $\mathbb{Z}_{\pi}:=\mathbb{Z}[\pi] /\left(\pi^{2}-1\right)$. We prove it by introducing a new grading by $\mathbb{Z}_{2} \times \mathbb{Z}$, in which the generators $X, Y$ and $Z$ have nontrivial degrees. We call it a splitting degree, because it decomposes the generalized Khovanov homology $\mathcal{H}(L)$ into a bunch of copies of $\mathcal{H}_{\pi}(L)$. An interesting feature of this degree is that it is not multiplicative with respect to the tensor product.

We use the new grading to construct an explicit duality isomorphism at the end of this paper. The main difficulty is that, after dualizing the complex, the roles of parameters $X$ and $Y$ are interchanged. In particular, the Frobenius-like algebra $A=\mathbb{k} v_{+} \oplus \mathbb{k} v_{-}$, associated to a circle, is different from its dual one. We overcome this with help from the splitting degree: not only do $v_{+}$and $v_{-}$have different degrees, but so do 
all generators of $A \otimes A$. Roughly speaking, we define a family of isomorphisms $A^{\otimes k} \stackrel{\cong}{\cong}\left(A^{*}\right)^{\otimes k}$ intertwining the algebra and coalgebra operations.

It is worth noticing that the generalized Khovanov homology $\mathcal{H}(L)$ is conjectured to extend projectively to link cobordisms [6, Conjecture 12.1]. The word "projective" means the assignment

$$
\{\text { link cobordisms }\} \rightarrow \text { \{chain maps }\}
$$

is defined only up to global invertible scalars. This would be enough to show that $\mathcal{H}(L)$ possesses the duality property similar to the one for $\mathcal{H}_{e v}(L)$, and, in particular, this would show indirectly the duality of odd Khovanov homology.

\section{Organization of the paper}

We first describe briefly the construction of the generalized Khovanov homology, including a discussion on chronological cobordisms and chronological TQFTs. The splitting degree is defined in Section 3 for both chronological cobordisms and modules over $\mathbb{k}$. In Section 4 we refine the generalized Khovanov complex to a graded complex, proving its invariance under Reidemeister moves. The last section contains the main results of this paper: the decomposition of the generalized Khovanov homology $\mathcal{H}(L)$ into a bunch of copies of the unifying one $\mathcal{H}_{\pi}(L)$, and the duality isomorphism between $\mathcal{H}\left(L^{!}\right)$and $\mathcal{H}(L)^{*}$, as well as the duality isomorphisms for the unifying and odd Khovanov homologies.

\section{Acknowledgements}

The authors are indebted to the referee for carefully reading the preliminary version of this paper and many remarks. The research was partially supported by the Columbia University Topology RTG grant DMS-0739392 and by the NCCR SwissMAP funded by the Swiss National Science Foundation.

\section{Basic definitions}

\subsection{Chronological cobordisms}

Definition 2.1 Let $W$ be a cobordism with a Riemann metric. A chronology on $W$ consists of a Morse function $h: W \rightarrow I$ that separates critical points, and a choice of an orientation of $E^{-}(p)$, the space of unstable directions in the gradient flow induced by $h$, at each critical point $p$. We require $h^{-1}(0)$ and $h^{-1}(1)$ to be the input and output of $W$ respectively. 
Chronological cobordisms admit two disjoint unions: the "left-then-right” $W \downarrow W^{\prime}$ and the "right-then-left" $W \downarrow \downarrow W^{\prime}$. Both are diffeomorphic to the standard disjoint union $W \sqcup W^{\prime}$, but to avoid a situation with two critical points at the same level, one has to pull all critical points of $W$ below $\frac{1}{2}$ and those of $W^{\prime}$ over $\frac{1}{2}$ (for $\downarrow$ ) or the other way (for $₫$ ):
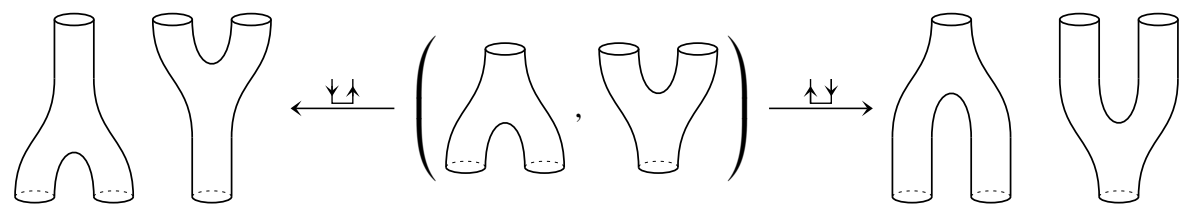

A standard argument from Morse theory shows that every 2-dimensional chronological cobordism can be built from six surfaces:

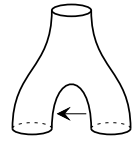

a merge

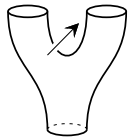

a split

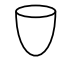

a birth

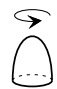

a positive death

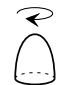

a negative

death

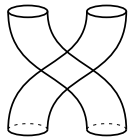

a twist

The little arrows visualize orientations of critical points. One merge and one split is sufficient, because the little arrow can be reversed by composing the cobordism with the twist.

Definition 2.2 Define the chronological degree deg $W \in \mathbb{Z} \times \mathbb{Z}$ of a chronological cobordism $W$ by setting

$$
\operatorname{deg} W=\text { (\#births - \#merges, \#deaths - \#splits). }
$$

The chronological degree is clearly additive with respect to composition of chronological cobordisms as well as to any of the disjoint unions.

Lemma 2.3 Given a chronological cobordism $W$ of degree $\operatorname{deg} W=(\alpha, \beta)$ with $k$ inputs and $\ell$ outputs, $\alpha+k=\beta+\ell$.

Proof Straightforward, by checking for generating cobordisms (1).

Choose a ring $\mathbb{k}:=\mathbb{Z}\left[X, Y, Z^{ \pm 1}\right] /\left(X^{2}=Y^{2}=1\right)$ and let $\mathbb{k}$ ChCob be a $\mathbb{k}$-linear category with finite disjoint unions of circles as objects, and $\mathbb{k}$-linear combinations of chronological cobordisms as morphisms, modulo the following chronological relations:
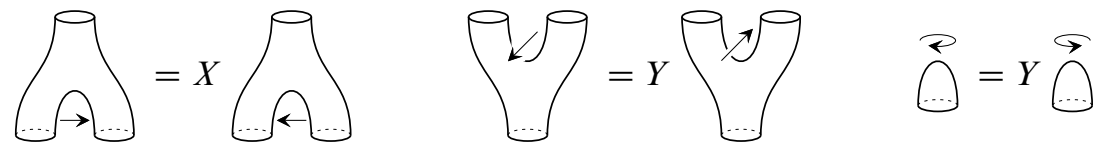
(4)
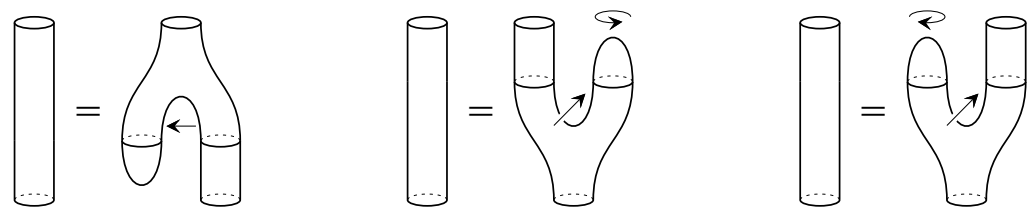

(5)
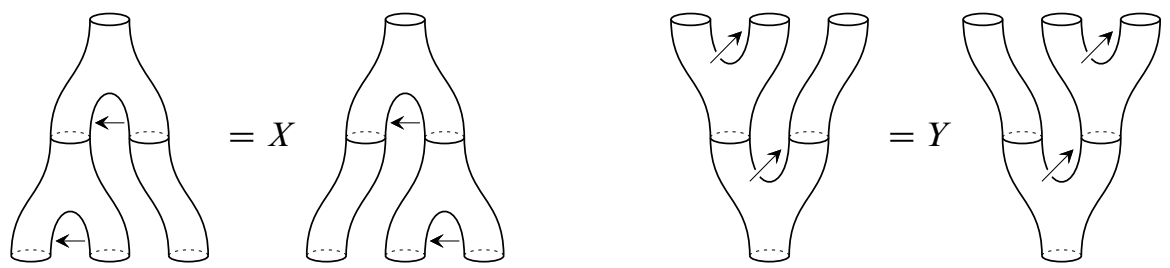

(6)

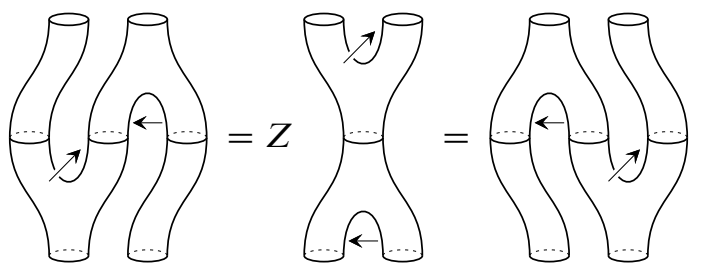

(7)

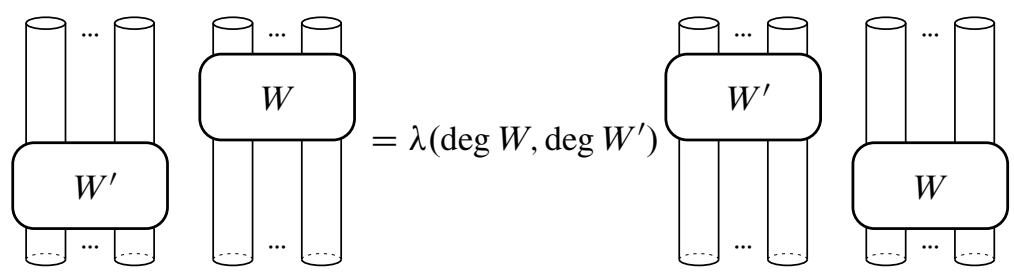

where $W$ and $W^{\prime}$ are any cobordisms and $\lambda\left(\alpha, \beta, \alpha^{\prime}, \beta^{\prime}\right)=X^{\alpha \alpha^{\prime}} Y^{\beta \beta^{\prime}} Z^{\alpha \beta^{\prime}-\alpha^{\prime} \beta}$. We proved in [6] the following non-degeneracy result for $\mathbb{k} \mathbf{C h C o b}$.

Proposition 2.4 Suppose $c W=0$ for a chronological cobordism $W$ and a nonzero $c \in \mathbb{k}$. Then $W$ has either positive genus or at least two closed components, and $c$ is divisible by $(X Y-1)$. In particular, cobordisms cannot be annihilated by monomials.

Remark 2.5 Given a ring homomorphism $\mathbb{k} \rightarrow R$ we define $R$ ChCob likewise. In particular, if we consider $\mathbb{Z}$ as a trivial $\mathbb{k}$-module, ie $X, Y$ and $Z$ act as the identity, $\mathbb{Z} \mathbf{C h C o b}$ is the linear extension of ordinary cobordisms.

\subsection{The generalized Khovanov complex}

We shall now briefly describe the construction of the generalized Khovanov complex from [6]. We encourage the reader to refer to Figure 1 frequently while reading this section; it illustrates the construction for the right-handed trefoil. 
Fix a link diagram $D$ and enumerate its crossings. Given a sequence $\xi=\left(\xi_{1}, \ldots, \xi_{n}\right)$, where $\xi_{i} \in\{0,1\}$ and $n$ is the number of crossings in $D$, let $D_{\xi}$ be a collection of circles obtained by resolving each crossing as illustrated below:

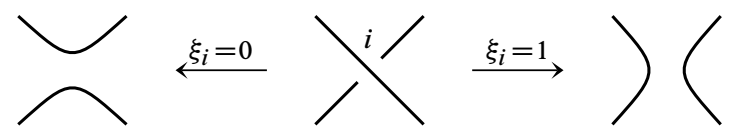

We call them type 0 and type 1 resolutions of a crossing. The diagrams $D_{\xi}$ decorate vertices of an $n$-dimensional cube $\mathcal{I}(D)$, called the cube of resolutions of $D$. Let $|\xi|:=\xi_{1}+\cdots+\xi_{n}$ be the weight of the vertex $\xi$. An edge $\zeta: \xi \rightarrow \xi^{\prime}$, oriented towards the vertex with higher weight, is decorated with a cobordism $D_{\zeta} \subset \mathbb{R}^{2} \times I$ that is a vertical surface except a small neighborhood of the resolution being changed, where a saddle, ? , is inserted. ${ }^{2}$ Decorate each crossing of $D$ with a small arrow that connects the two arcs in type 0 resolution; these arrows determine uniquely orientations of saddle points of the cobordisms $D_{\zeta}$, so that $\mathcal{I}(D)$ can be regarded as a diagram in the category $\mathbb{k} \mathbf{C h C o b}$.

The cube $\mathcal{I}(D)$ does not commute in general. Nevertheless, there is a cubical cocycle $\psi \in C^{2}\left(I^{n} ; \mathbb{k}^{*}\right)$ such that for every face $S$ of the form

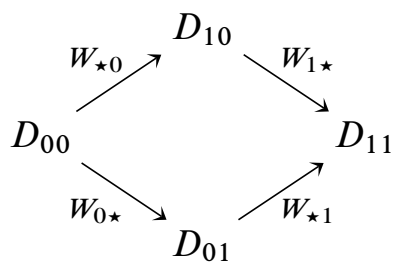

the twisted commutativity $W_{1 \star} W_{\star 0}=\psi(S) W_{\star 1} W_{0 \star}$ holds. If $\delta \epsilon=-\psi$ for a cubical cochain $\epsilon \in C^{1}\left(I^{n} ; \mathbb{k}^{*}\right)$, the corrected cube $\mathcal{I}^{\epsilon}(D)$, in which each cobordism $D_{\zeta}$ is multiplied by $\epsilon(\zeta)$, anticommutes. We call such a 1-cochain a sign assignment.

The generalized Khovanov complex is constructed in Mat $(\mathbb{k} \mathbf{C h C o b})$, the additive closure of the category $\mathbb{k} \mathbf{C h C o b : ~ o b j e c t s ~ a r e ~ f i n i t e ~ s e q u e n c e s ~ ( v e c t o r s ) ~ o f ~ 1 - m a n i f o l d s , ~}$ and morphisms are matrices with linear combinations of chronological cobordisms as entries. A direct sum in $\operatorname{Mat}(\mathbb{k} \mathbf{C h C o b})$ is realized by concatenation of sequences.

Definition 2.6 Let $D$ be a link diagram with enumerated and oriented crossings. Given a sign assignment $\epsilon$ for the cube $\mathcal{I}(D)$ we define the generalized Khovanov

${ }^{2}$ In Figure 1 we use the surgery description of cobordisms: the input circles together with an arc, a surgery along which results in the output circles. The arc is oriented, inducing an orientation of the saddle. A $3 \mathrm{D}$ picture of one cobordism is provided in the left bottom corner of the picture. 


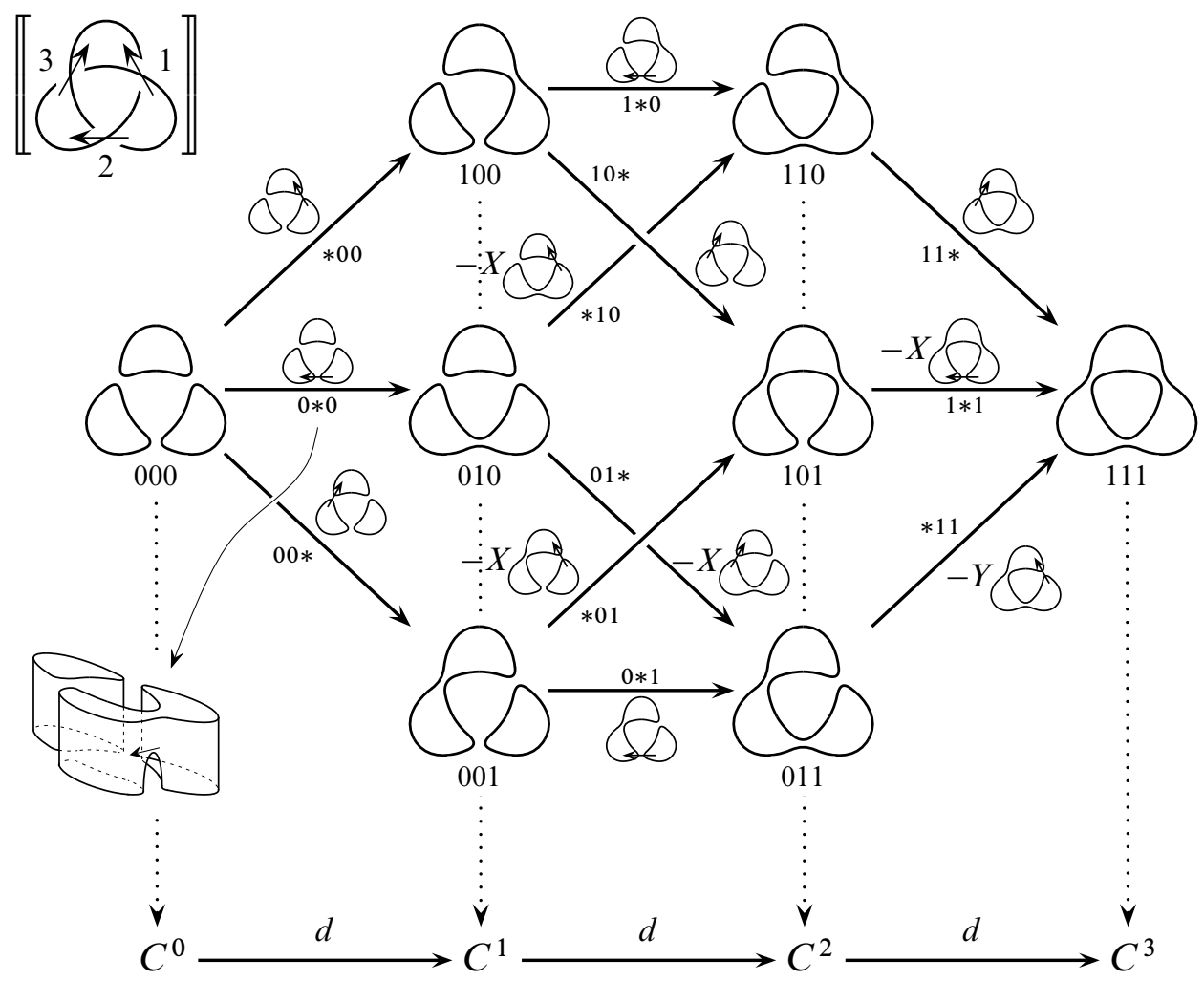

Figure 1: The cube of resolutions and the generalized Khovanov complex for the right-handed trefoil. The coefficients $X$ and $Y$ are part of a sign assignment.

bracket as the chain complex $\llbracket D \rrbracket_{\epsilon}$ in the category $\operatorname{Mat}(\mathbb{k} \mathbf{C h C o b})$ with

$$
\llbracket D \rrbracket_{\epsilon}^{i}:=\bigoplus_{|\xi|=i} D_{\xi},\left.\quad d^{i}\right|_{D_{\xi}}:=\sum_{\zeta: \xi \rightarrow \xi^{\prime}} \epsilon(\zeta) D_{\zeta} .
$$

The generalized Khovanov complex $K h(D)$ is obtained from $\llbracket D \rrbracket_{\epsilon}$ by shifting it to the left by the number of negative crossings, ie $K h(D)^{i}:=\llbracket D \rrbracket_{\epsilon}^{i+n_{-}}$.

Remark 2.7 The generalized Khovanov bracket and complex admits an integral grading induced from the $(\mathbb{Z} \times \mathbb{Z})$-grading of chronological cobordisms [6, Section 5]. We skip the details, as this degree does not play any role in this paper.

Theorem 2.8 [6, Theorem 7.1] The homotopy type of the generalized Khovanov complex $K h(D)$ is a link invariant, when regarded as a complex in the category Mat $(\mathbb{k} \mathbf{C h C o b})$ modulo the following three relations in which all deaths are negative: 
(S)

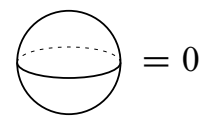

(T)

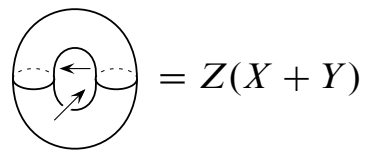

$(4 \mathrm{Tu})$

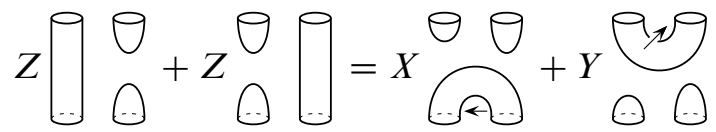

The proof of the theorem can be found in [6, Section 7]. It is revisited in Section 4, where we inspect the chain maps involved in the proof against the new grading described in the next section.

\subsection{Chronological TQFTs and homology}

Consider the category $\operatorname{Mod}_{\mathbb{k}}$ of $\mathbb{k}$-modules graded by the group $\mathbb{Z} \times \mathbb{Z}$. We redefine the tensor product for homomorphisms by setting for homogeneous maps $f$ and $g$

$$
(f \otimes g)(m \otimes n):=\lambda(\operatorname{deg} g, \operatorname{deg} m) f(m) \otimes g(n),
$$

where $\lambda\left(\alpha, \beta, \alpha^{\prime}, \beta^{\prime}\right)=X^{\alpha \alpha^{\prime}} Y^{\beta \beta^{\prime}} Z^{\alpha \beta^{\prime}-\alpha^{\prime} \beta}$ is defined as for $\mathbb{k} \mathbf{C h C o b}$. One checks directly that

$$
\left(f^{\prime} \otimes g^{\prime}\right) \circ(f \otimes g)=\lambda\left(\operatorname{deg} g^{\prime}, \operatorname{deg} f\right)\left(f^{\prime} \circ f\right) \otimes\left(g^{\prime} \circ g\right) .
$$

Hence, $\operatorname{Mod}_{\mathbb{K}}$ is a graded tensor category in the sense of [6, Definition 10.1]. There is a symmetry $\tau_{M, N}: M \otimes N \rightarrow N \otimes M$ given by $\tau_{M, N}(m \otimes n)=\lambda(\operatorname{deg} m, \operatorname{deg} n) n \otimes m$ for homogeneous elements $m \in M$ and $n \in N$.

Definition 2.9 A chronological TQFT is a functor $\mathcal{F}$ : $\mathbb{k} \mathbf{C h C o b} \rightarrow \operatorname{Mod}_{\mathbb{k}}$ that preserves the $\mathbb{Z} \times \mathbb{Z}$ grading, and which maps the "right-then-left" disjoint union $\downarrow \downarrow$ into the graded tensor product $\otimes$ and the twist $\&$ into the symmetry $\tau$.

We defined in [6, Example 10.7] a chronological TQFT $\mathcal{F}: \mathbb{k}_{\mathrm{k}} \mathbf{C h C o b} \rightarrow \mathbf{M o d}_{\mathbb{k}}$ that maps a circle to the module $A$ freely degenerated by $v_{+}$in degree $(1,0)$ and $v_{-}$in degree $(0,-1)$, and generating cobordisms to the following maps:

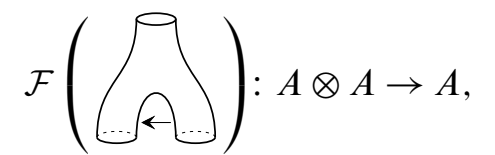

$$
\left\{\begin{array}{l}
v_{+} \otimes v_{+} \mapsto v_{+} \\
v_{-} \otimes v_{-} \mapsto 0
\end{array}\right.
$$$$
\begin{aligned}
& v_{+} \otimes v_{-} \mapsto v_{-} \\
& v_{-} \otimes v_{+} \mapsto X Z v_{-}
\end{aligned}
$$ 


$$
\begin{array}{rlrl}
\mathcal{F}(\underbrace{}_{y}): A \rightarrow A \otimes A, & & \left\{\begin{array}{l}
v_{+} \mapsto v_{-} \otimes v_{+}+Y Z v_{+} \otimes v_{-}, \\
v_{-} \mapsto v_{-} \otimes v_{-},
\end{array}\right. \\
\mathcal{F}(\bigoplus): \mathbb{k} \rightarrow A, & 1 \mapsto v_{+}, \\
\mathcal{F}(\bigodot): A \rightarrow \mathbb{k}, & v_{+} \mapsto 0, \quad v_{-} \mapsto 1 .
\end{array}
$$

It is easy to check that $\mathcal{F}$ preserves the $(\mathbb{Z} \times \mathbb{Z})$-grading. Likewise, given a ring homomorphism $\mathbb{k} \rightarrow R$, we define a TQFT $\mathcal{F}_{R}: R \mathbf{C h C o b} \rightarrow \operatorname{Mod}_{R}$ such that $\mathcal{F}_{R}(\bigcirc)=A \otimes R$.

Definition 2.10 The generalized Khovanov homology $\mathcal{H}(L)$ of a link $L$ is the homology of the chain complex $\mathcal{F} K h(D)$, where $D$ is a diagram of $L$. Given a $\mathbb{k}$-module $M$ we write $\mathcal{H}(L ; M)$ for the homology of the complex $\mathcal{F} K h(D) \otimes M$.

Example 2.11 We distinguish two out of eight $\mathbb{k}$-algebra structures on the ring $\mathbb{Z}$ :

- $\mathbb{Z}_{e v}$, on which all $X, Y$ and $Z$ act trivially, and

- $\mathbb{Z}_{\text {odd }}$, on which $X$ and $Z$ act trivially, but $Y$ acts as multiplication by -1 .

We have shown in $[6$, Proposition 10.8$]$ that $\mathcal{H}\left(L ; \mathbb{Z}_{e v}\right)$ and $\mathcal{H}\left(L ; \mathbb{Z}_{\text {odd }}\right)$ are the even and odd Khovanov homology respectively. Both are specializations of $\mathcal{H}\left(L ; \mathbb{Z}_{\pi}\right)$, where $\mathbb{Z}_{\pi}=\mathbb{Z}[\pi] /\left(\pi^{2}-1\right)$ is a $\mathbb{k}$-module, on which both $X$ and $Z$ act as the identity, and $Y$ as the multiplication by $\pi$.

\section{The new grading}

\subsection{The grading on chronological cobordisms}

The category $\mathbb{k} \mathbf{C h C o b}$ of chronological cobordisms admits an additional grading by the group $\mathbb{Z}_{2} \times \mathbb{Z}$, which we shall refer to as the splitting degree for a reason explained later. It takes the following values on the generating cobordisms:

$$
\begin{aligned}
& \operatorname{sdeg}(\Omega)=\left[\begin{array}{l}
0 \\
0
\end{array}\right], \quad \operatorname{sdeg}(\Theta)=\left[\begin{array}{l}
0 \\
0
\end{array}\right],
\end{aligned}
$$

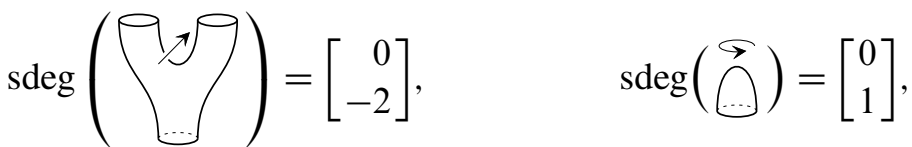




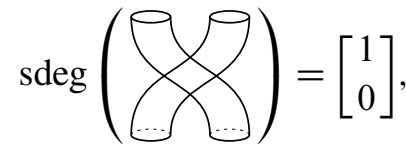

$$
\operatorname{sdeg}(\stackrel{P}{\bigodot})=\left[\begin{array}{l}
1 \\
1
\end{array}\right]
$$

and the coefficient ring $\mathbb{k}$ is graded itself with

$$
\operatorname{sdeg} X=\operatorname{sdeg} Y=\left[\begin{array}{l}
1 \\
0
\end{array}\right], \quad \operatorname{sdeg} Z=\left[\begin{array}{r}
0 \\
-1
\end{array}\right] .
$$

We use the vertical notation for elements of $\mathbb{Z}_{2} \times \mathbb{Z}$ to distinguish it from the chronological degree. This degree is not additive with respect to the disjoint union; instead we set

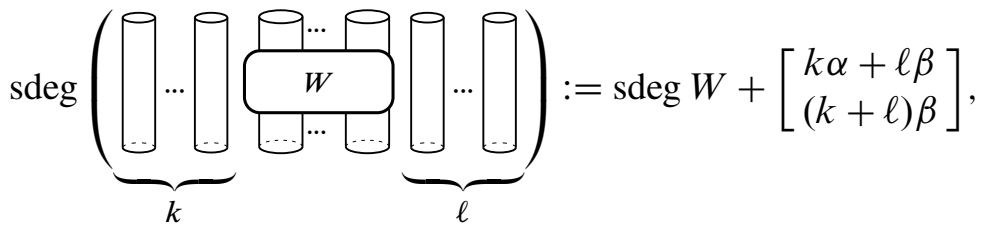

assuming $\operatorname{deg} W=(\alpha, \beta)$. The above formula is clearly additive with respect to composition of cobordisms, and it is coherent with the symmetry:

(20) $\quad$ sdeg

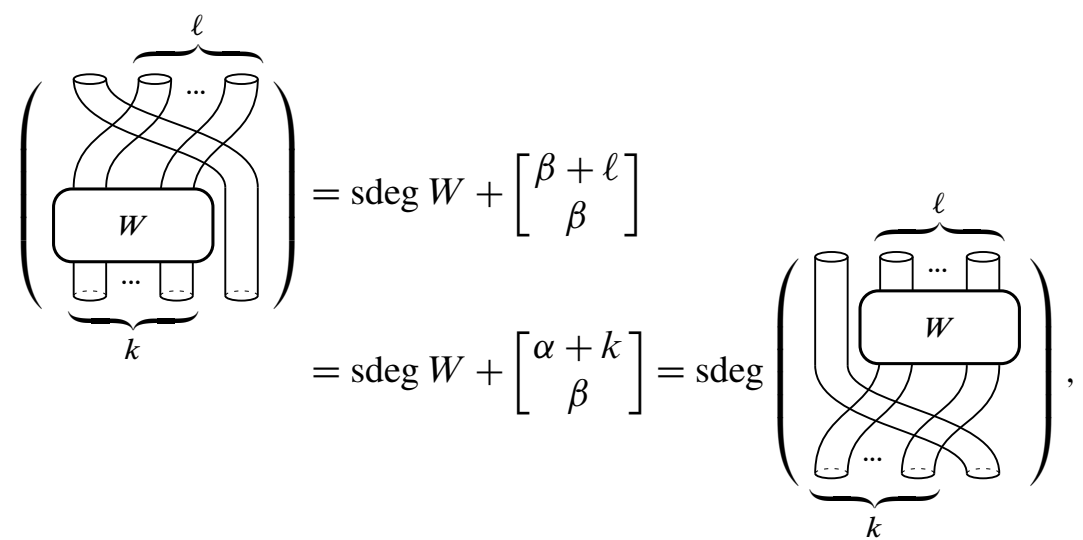

where $\operatorname{deg} W=(\alpha, \beta)$, and the equality $\alpha+k=\beta+\ell$ follows from Lemma 2.3.

Proposition 3.1 The chronological relations are homogeneous with respect to the splitting degree.

Proof Creation and annihilation do not change the degree, as we can directly compute:

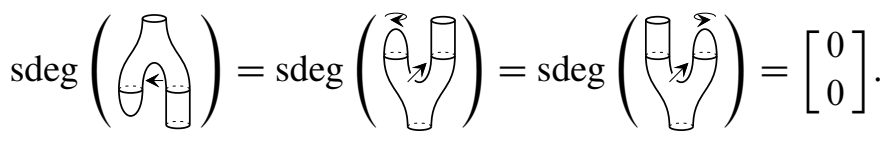


Choose cobordisms $W_{i}: k_{i} \mathbb{S}^{1} \rightarrow \ell_{i} \mathbb{S}^{1}$ for $i=1$, 2. If $\operatorname{deg} W_{i}=\left(\alpha_{i}, \beta_{i}\right)$, we have

$$
\begin{aligned}
& \operatorname{sdeg}\left(W_{1} \triangleleft W_{2}\right)=\operatorname{sdeg}\left(W_{1} \sqcup C_{\ell_{2} \mathbb{S}^{1}}\right)+\operatorname{sdeg}\left(C_{k_{1} \mathbb{S}^{1}} \sqcup W_{2}\right), \\
& \operatorname{sdeg}\left(W_{1} \Downarrow W_{2}\right)=\operatorname{sdeg}\left(W_{1} \sqcup C_{k_{2} \mathbb{S}^{1}}\right)+\operatorname{sdeg}\left(C_{\ell_{1} \mathbb{S}^{1}} \sqcup W_{2}\right),
\end{aligned}
$$

where $C_{n \mathbb{S}^{1}}$ is a disjoint union of $n$ vertical tubes. Using the formula (19) we compute

$$
\begin{aligned}
\operatorname{sdeg}\left(W_{1} \hookleftarrow \Downarrow W_{2}\right)-\operatorname{sdeg}\left(W_{1} \Downarrow W_{2}\right) & =\left[\begin{array}{l}
\left(\ell_{2}-k_{2}\right) \beta_{1} \\
\left(\ell_{2}-k_{2}\right) \beta_{1}
\end{array}\right]+\left[\begin{array}{c}
\left(k_{1}-\ell_{1}\right) \alpha_{2} \\
\left(k_{1}-\ell_{1}\right) \beta_{2}
\end{array}\right] \\
& =\left[\begin{array}{l}
\beta_{1} \beta_{2}+\alpha_{1} \alpha_{2} \\
\beta_{1} \alpha_{2}-\alpha_{1} \beta_{2}
\end{array}\right] \\
& =\operatorname{sdeg}\left(X^{\alpha_{1} \alpha_{2}} Y^{\beta_{1} \beta_{2}} Z^{\alpha_{1} \beta_{2}-\beta_{1} \alpha_{2}}\right),
\end{aligned}
$$

which shows that $W_{1} \downarrow \downarrow W_{2}$ and $\lambda\left(\operatorname{deg} W_{1}, \operatorname{deg} W_{2}\right) W_{1} \downarrow \uparrow W_{2}$ have the same degree. The remaining chronological relations are easily checked by hand:

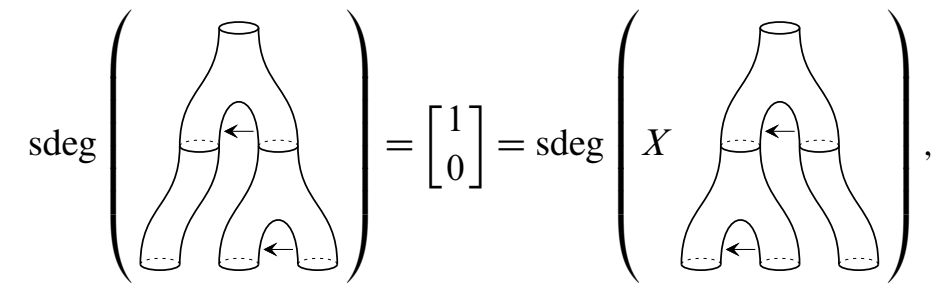

(26)
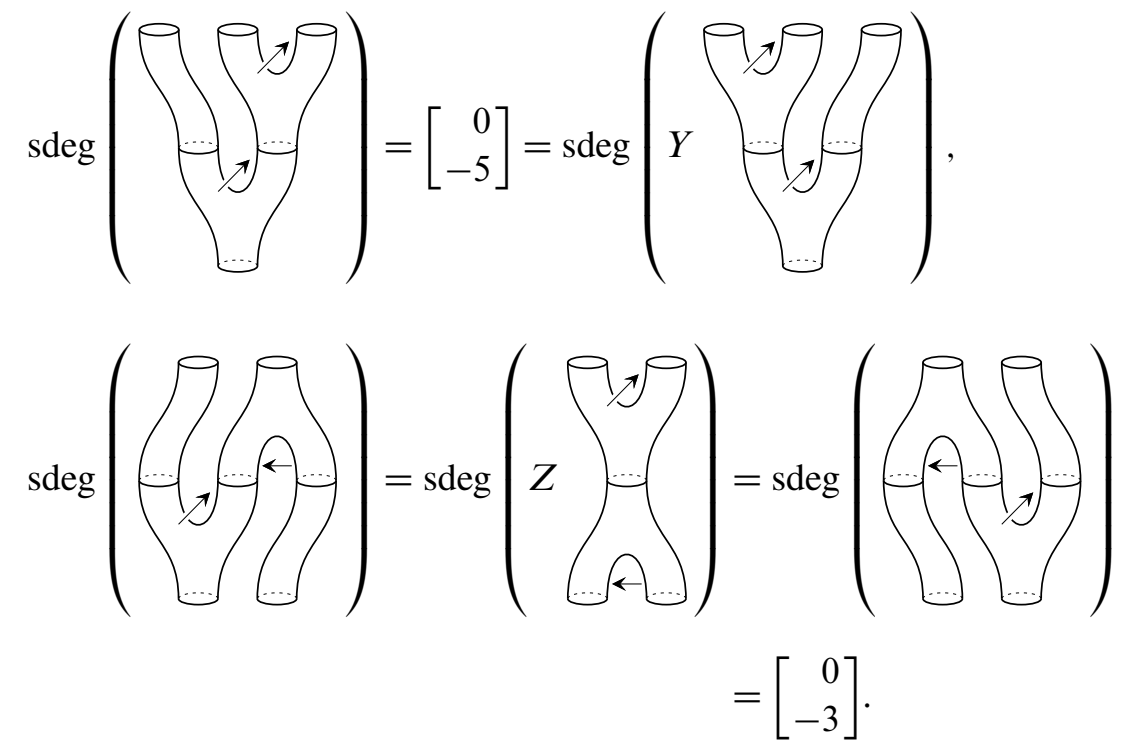
Observation 3.2 The splitting degree decomposes the module of morphisms into

$$
\operatorname{Mor}\left(\Sigma, \Sigma^{\prime}\right):=\bigoplus_{(k, \ell) \in \mathbb{Z}_{2} \times \mathbb{Z}} \operatorname{Mor}_{(k, \ell)}\left(\Sigma, \Sigma^{\prime}\right),
$$

where each summand is isomorphic to the submodule of degree-preserving maps. Indeed,

$$
\operatorname{sdeg}\left(X^{a} Z^{b} f\right)=\left[\begin{array}{l}
0 \\
0
\end{array}\right] \quad \text { if } \quad \operatorname{sdeg} f=\left[\begin{array}{l}
a \\
b
\end{array}\right]
$$

We make $\mathbb{k} \mathbf{C h C o b}$ a graded category by replacing its objects with symbols $\Sigma\{a, b\}$, where $\Sigma$ is an object of $\mathbb{k} \mathbf{C h C o b}$ and $(a, b) \in \mathbb{Z}_{2} \times \mathbb{Z}$. Thinking of $\{a, b\}$ as a degree shift operation, the module of morphisms is given as a direct sum

$$
\operatorname{Mor}\left(\Sigma\{a, b\}, \Sigma^{\prime}\left\{a^{\prime}, b^{\prime}\right\}\right):=\bigoplus_{(k, \ell) \in \mathbb{Z}_{2} \times \mathbb{Z}} \operatorname{Mor}_{\left(k+a-a^{\prime}, \ell+b-b^{\prime}\right)}\left(\Sigma, \Sigma^{\prime}\right),
$$

ie a homogeneous morphism $f \in \operatorname{Mor}_{(k, \ell)}\left(\Sigma, \Sigma^{\prime}\right)$, when regarded as a morphism $f: \Sigma\{a, b\} \rightarrow \Sigma^{\prime}\left\{a^{\prime}, b^{\prime}\right\}$, has degree

$$
\operatorname{sdeg} f=\left[\begin{array}{c}
k-a+a^{\prime} \\
\ell-b+b^{\prime}
\end{array}\right] .
$$

We write $\mathbb{k} \mathbf{C h C o b _ { 0 }}$ for the subcategory of degree-preserving morphisms.

\subsection{The grading on modules}

Recall that the ring $\mathbb{k}$ is graded by $\mathbb{Z}_{2} \times \mathbb{Z}$, with

$$
\operatorname{sdeg} X=\operatorname{sdeg} Y=\left[\begin{array}{l}
1 \\
0
\end{array}\right], \quad \operatorname{sdeg} Z=\left[\begin{array}{r}
0 \\
-1
\end{array}\right] .
$$

Hereafter let $\operatorname{Mod}_{\mathbb{k}}$ stand for the category of modules with a compatible $\left(\mathbb{Z}_{2} \times \mathbb{Z}\right)$ grading, in addition to the $(\mathbb{Z} \times \mathbb{Z})$-grading, and write $\operatorname{Mod}_{\mathbb{k}, 0}$ for the subcategory formed by maps that preserve the new degree. Again, the new grading is not additive with respect to the tensor product, but instead we set

$$
\operatorname{sdeg}(m \otimes n):=\operatorname{sdeg}(m)+\operatorname{sdeg}(n)+\left[\begin{array}{l}
\beta\|n\| \\
\beta\|n\|
\end{array}\right]
$$

for $m \in M$ and $n \in N$ homogeneous with respect to both degrees, where $\operatorname{deg} m=$ $(\alpha, \beta)$ and $\|n\|$ is the weight of $n$ : the difference of the two components of deg, eg $\|m\|=\alpha-\beta$. The name is motivated by the behavior of the symmetry isomorphism: it is homogeneous only when restricted to submodules supported in a single weight. 
Lemma 3.3 The associator $\left(M_{1} \otimes M_{2}\right) \otimes M_{3} \rightarrow M_{1} \otimes\left(M_{2} \otimes M_{3}\right)$ preserves the splitting degree. Moreover, if $M_{1}$ and $M_{2}$ are supported in weights $w_{1}$ and $w_{2}$ respectively, the symmetry $\tau: M_{1} \otimes M_{2} \rightarrow M_{2} \otimes M_{1}$ is homogeneous of degree

$$
\operatorname{sdeg} \tau=\left[\begin{array}{c}
w_{1} w_{2} \\
0
\end{array}\right]
$$

Proof Choose elements $m_{i} \in M_{i}, i=1,2,3$, with $\mathbb{Z} \times \mathbb{Z}$ degrees $\operatorname{deg} m_{i}=\left(\alpha_{i}, \beta_{i}\right)$. Using formula (30) we compute

$\operatorname{sdeg}\left(\left(m_{1} \otimes m_{2}\right) \otimes m_{3}\right)$

$$
\begin{aligned}
& =\operatorname{sdeg}\left(m_{1} \otimes m_{2}\right)+\operatorname{sdeg}\left(m_{3}\right)+\left[\begin{array}{l}
\left(\beta_{1}+\beta_{2}\right)\left\|m_{3}\right\| \\
\left(\beta_{1}+\beta_{2}\right)\left\|m_{3}\right\|
\end{array}\right] \\
& =\operatorname{sdeg}\left(m_{1}\right)+\operatorname{sdeg}\left(m_{2}\right)+\operatorname{sdeg}\left(m_{3}\right)+\left[\begin{array}{l}
\beta_{1}\left\|m_{2}\right\|+\beta_{1}\left\|m_{3}\right\|+\beta_{2}\left\|m_{3}\right\| \\
\beta_{1}\left\|m_{2}\right\|+\beta_{1}\left\|m_{3}\right\|+\beta_{2}\left\|m_{3}\right\|
\end{array}\right] \\
& =\operatorname{sdeg}\left(m_{1}\right)+\operatorname{sdeg}\left(m_{2} \otimes m_{3}\right)+\left[\begin{array}{l}
\beta_{1}\left\|m_{2} \otimes m_{3}\right\| \\
\beta_{1}\left\|m_{2} \otimes m_{3}\right\|
\end{array}\right] \\
& =\operatorname{sdeg}\left(m_{1} \otimes\left(m_{2} \otimes m_{3}\right)\right) .
\end{aligned}
$$

For the second statement we compute $\tau\left(m_{1} \otimes m_{2}\right)=X^{\alpha_{1} \alpha_{2}} Y^{\beta_{1} \beta_{2}} Z^{\alpha_{1} \beta_{2}-\beta_{1} \alpha_{2}} m_{2} \otimes m_{1}$. Then,

$\operatorname{sdeg}\left(\tau\left(m_{1} \otimes m_{2}\right)\right)-\operatorname{sdeg}\left(m_{1} \otimes m_{2}\right)$

$$
\begin{aligned}
& =\left[\begin{array}{c}
\alpha_{1} \alpha_{2}+\beta_{1} \beta_{2} \\
\beta_{1} \alpha_{2}-\alpha_{1} \beta_{2}
\end{array}\right]+\operatorname{sdeg}\left(m_{2} \otimes m_{1}\right)-\operatorname{sdeg}\left(m_{1} \otimes m_{2}\right) \\
& =\left[\begin{array}{c}
\alpha_{1} \alpha_{2}+\beta_{1} \beta_{2} \\
\beta_{1} \alpha_{2}-\alpha_{1} \beta_{2}
\end{array}\right]+\left[\begin{array}{l}
\beta_{2} w_{1} \\
\beta_{2} w_{1}
\end{array}\right]-\left[\begin{array}{l}
\beta_{1} w_{2} \\
\beta_{1} w_{2}
\end{array}\right] \\
& =\left[\begin{array}{c}
\left(\alpha_{1}-\beta_{1}\right)\left(\alpha_{2}-\beta_{2}\right) \\
0
\end{array}\right] \\
& =\left[\begin{array}{c}
w_{1} w_{2} \\
0
\end{array}\right],
\end{aligned}
$$

which ends the proof.

Lemma 3.4 Choose a homogeneous map $f: M \rightarrow N$ of degree $\operatorname{deg} f=(\alpha, \beta)$, and two modules $M^{\prime}$ and $M^{\prime \prime}$ supported in weights $k$ and $\ell$ respectively. Then

$$
\operatorname{sdeg}\left(\operatorname{id}_{M^{\prime}} \otimes f \otimes \operatorname{id}_{M^{\prime \prime}}\right)=\operatorname{sdeg} f+\left[\begin{array}{l}
k \alpha+\ell \beta \\
(k+\ell) \beta
\end{array}\right] .
$$

In particular, the graded tensor product relation (11) is graded. 
Proof Pick homogeneous elements $m_{1} \in M^{\prime}, m_{2} \in M$ and $m_{3} \in M^{\prime \prime}$, each of $\mathbb{Z} \times \mathbb{Z}$ degree $\operatorname{deg}\left(m_{i}\right)=\left(\alpha_{i}, \beta_{i}\right)$. Then

$\operatorname{sdeg}\left((\mathrm{id} \otimes f \otimes \mathrm{id})\left(m_{1} \otimes m_{2} \otimes m_{3}\right)\right)-\operatorname{sdeg}\left(m_{1} \otimes m_{2} \otimes m_{3}\right)$

$$
\begin{aligned}
& =\operatorname{sdeg}\left(X^{\alpha_{1} \alpha} Y^{\beta_{1} \beta} Z^{\beta_{1} \alpha-\alpha_{1} \beta} m_{1} \otimes f\left(m_{2}\right) \otimes m_{3}\right)-\operatorname{sdeg}\left(m_{1} \otimes m_{2} \otimes m_{3}\right) \\
& =\operatorname{sdeg} f+\left[\begin{array}{c}
\beta_{1} \beta+\alpha_{1} \alpha \\
\beta \alpha_{1}-\beta_{1} \alpha
\end{array}\right]+\left[\begin{array}{c}
\beta_{1}(\alpha-\beta)+\beta\left(\alpha_{3}-\beta_{3}\right) \\
\beta_{1}(\alpha-\beta)+\beta\left(\alpha_{3}-\beta_{3}\right)
\end{array}\right] \\
& =\operatorname{sdeg} f+\left[\begin{array}{l}
k \alpha+\ell \beta \\
(k+\ell) \beta
\end{array}\right] .
\end{aligned}
$$

The last statement follows from a direct computation, as in Proposition 3.1.

The generators of the module $A$ have weights $\left\|v_{+}\right\|=\left\|v_{-}\right\|=1$, implying that

$$
\operatorname{sdeg}\left(\operatorname{id}_{A \otimes k} \otimes f \otimes \operatorname{id}_{A} \otimes \ell\right)=\operatorname{sdeg} f+\left[\begin{array}{l}
k \alpha+\ell \beta \\
(k+\ell) \beta
\end{array}\right],
$$

which is similar to the formula (19). We define the splitting degree on $A$ by setting

$$
\operatorname{sdeg} v_{+}=\left[\begin{array}{l}
0 \\
0
\end{array}\right], \quad \operatorname{sdeg} v_{-}=\left[\begin{array}{r}
1 \\
-1
\end{array}\right] .
$$

Table 1 contains degrees of generators of $A^{\otimes 2}$.

\begin{tabular}{r|cccc}
\hline generator & $v_{+} \otimes v_{+}$ & $v_{+} \otimes v_{-}$ & $v_{-} \otimes v_{+}$ & $v_{-} \otimes v_{-}$ \\
\hline $\operatorname{deg}$ & $(2,0)$ & $(1,-1)$ & $(1,-1)$ & $(0,-2)$ \\
$\operatorname{sdeg}$ & {$\left[\begin{array}{l}0 \\
0\end{array}\right]$} & {$\left[\begin{array}{r}1 \\
-1\end{array}\right]$} & {$\left[\begin{array}{r}0 \\
-2\end{array}\right]$} & {$\left[\begin{array}{r}1 \\
-3\end{array}\right]$} \\
\hline
\end{tabular}

Table 1: Degrees of generators of the second power of $A$

Lemma 3.5 A generator $v=v_{k} \otimes \cdots \otimes v_{1} \in A^{\otimes k}$ is homogeneous of degree

$$
\operatorname{sdeg} v=\left[\begin{array}{l}
a \\
a
\end{array}\right]
$$

with $a=-\sum_{v_{i}=v_{-}} i$.

Proof It follows from an easy induction argument and is left to the reader.

Proposition 3.6 The functor $\mathcal{F}: \mathbb{k} \mathbf{C h C o b} \rightarrow \operatorname{Mod}_{\mathbb{k}}$ preserves the splitting degree. 
Proof In view of Lemma 3.3 and formula (32) it is enough to check that the four maps (12)-(15) have the same degrees as the corresponding cobordisms. This follows directly from the expressions for these maps and Lemma 3.5.

\section{Invariance revisited}

We shall introduce the splitting degree to the generalized Khovanov complex. Choose a link diagram $D$ and construct its cube of resolutions $\mathcal{I}^{\epsilon}(D)$ corrected by a certain sign assignment $\epsilon$. For every vertex $\xi$ choose a directed path to $\xi$ originating at the initial vertex $(0, \ldots, 0)$, and denote by $W_{\xi}$ the cobordism the path encodes. ${ }^{3}$ Shift vertices of the cube by degrees of the cobordisms $W_{\xi}$ :

$$
\mathcal{I}^{\epsilon}(D)(\xi):=D_{\xi}\left\{\operatorname{sdeg} W_{\xi}\right\} .
$$

As the faces anticommute, sdeg $W_{\xi}$ does not depend on the path chosen. This modification results in a cube in the category $\mathbb{k} \mathbf{C h} \mathbf{C o b} b_{0}$, ie all morphisms preserve the splitting degree. In particular, the differential in the generalized Khovanov bracket $\llbracket D \rrbracket_{\epsilon}$ is a degree-preserving morphism.

Theorem 4.1 The homotopy type of the graded generalized Khovanov complex is a link invariant modulo the relations $(\mathrm{S}),(\mathrm{T})$, and $(4 \mathrm{Tu})$. In particular, the generalized Khovanov homology $\mathcal{H}(L)$ admits a $\left(\mathbb{Z}_{2} \times \mathbb{Z}\right)$-grading coherent with the action of $\mathbb{k}$.

The proof will be given in a few steps. We first check that the mentioned relations are coherent with the new grading.

Lemma 4.2 The relations (S), (T) and (4Tu) from Theorem 2.8 are homogeneous with respect to the splitting degree.

Proof The statement obviously holds for the relation (S), and for (T) we compute

$$
\begin{aligned}
\operatorname{sdeg}(\bigoplus) & =\operatorname{sdeg}(\stackrel{P}{\Omega})+\operatorname{sdeg}(\Omega))+\operatorname{sdeg}(\Omega \pi \rho)+\operatorname{sdeg}(\theta) \\
& =\left[\begin{array}{r}
1 \\
-1
\end{array}\right],
\end{aligned}
$$

\footnotetext{
${ }^{3}$ The path is empty if $\xi=(0, \ldots, 0)$, in which case $W_{\xi}$ is the cylinder $D_{\xi} \times I$.
} 
which is the splitting degree of $Z(X+Y)$. Finally, a direct computation shows that each term of (4Tu) has degree $\left[\begin{array}{l}0 \\ 1\end{array}\right]$. For instance, using (19) we compute

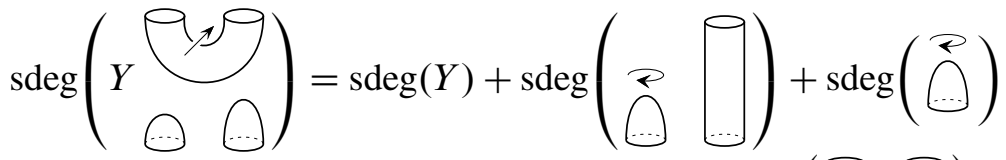

$$
\begin{aligned}
& +\operatorname{sdeg}(\theta)+\operatorname{sdeg}((\pi)) \\
& =\left[\begin{array}{l}
1 \\
0
\end{array}\right]+\left[\begin{array}{l}
1+1 \\
1+1
\end{array}\right]+\left[\begin{array}{l}
1 \\
1
\end{array}\right]+\left[\begin{array}{l}
0 \\
0
\end{array}\right]+\left[\begin{array}{c}
0 \\
-2
\end{array}\right] \\
& =\left[\begin{array}{l}
0 \\
1
\end{array}\right] \text {, }
\end{aligned}
$$

and similarly for the other terms.

We shall show that the homotopy type of the generalized Khovanov chain complex is invariant up to an overall degree shift. A priori this shows only that the relative grading is preserved, ${ }^{4}$ but then one can easily deduce invariance of the absolute grading from the following lemma (see also Observation 3.2).

Lemma 4.3 Suppose there is a homotopy equivalence $f: \llbracket D \rrbracket \stackrel{\simeq}{\longrightarrow} \llbracket D^{\prime} \rrbracket$ that is homogeneous with respect to the splitting degree. Then there exists a homotopy equivalence $\llbracket D \rrbracket \stackrel{\simeq}{\longrightarrow} \llbracket D^{\prime} \rrbracket$ of splitting degree 0 .

Proof Let $\operatorname{sdeg} f=\left[\begin{array}{l}a \\ b\end{array}\right]$. Then $X^{a} Z^{b} f$ is the desired chain map.

Hence, our goal is to show that all isomorphisms involved in the proof of invariance from [6] are homogeneous. We begin by inspecting the extra choices made in the construction of the generalized Khovanov bracket. The key tool is the following result.

Lemma 4.4 Suppose there is a commutative square in $\mathbb{k}$ ChCob

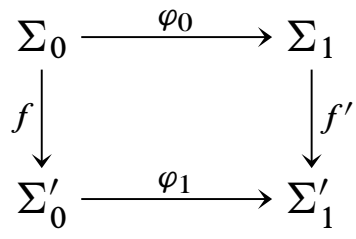

\footnotetext{
${ }^{4}$ A chain map $f$ preserves the relative grading if $\operatorname{sdeg} u-\operatorname{sdeg} v=\operatorname{sdeg} f(u)-\operatorname{sdeg} f(v)$, but $\operatorname{sdeg} u$ may be different from sdeg $f(u)$ in general.
} 
where each morphism is a chronological cobordism scaled by an invertible element from $\mathbb{k}$. If each $\varphi_{i}$ is graded with respect to the splitting degree, ie $\operatorname{sdeg} \varphi_{i}=\left[\begin{array}{l}0 \\ 0\end{array}\right]$, then $\operatorname{sdeg} f=\operatorname{sdeg} f^{\prime}$.

Proof It is enough to show that the composition $\varphi_{1} f=f^{\prime} \varphi_{0}$ does not vanish. This follows from Proposition 2.4.

Sign assignments Given two sign assignments $\epsilon_{1}, \epsilon_{2}$ of the cube $\mathcal{I}(D)$, the corrected cubes $\mathcal{I}^{\epsilon_{1}}(D), \mathcal{I}^{\epsilon_{2}}(D)$ are isomorphic via a family of morphisms $f_{\xi}:=v(\xi)$ id, where $v \in C^{0}\left(I^{n} ; \mathbb{k}^{*}\right)$ is a cochain such that $\epsilon_{2}=\delta v \cdot \epsilon_{1}$. Since no $v(\xi)$ vanishes, it follows from Lemma 4.4 that each $f_{\xi}$ is a homogeneous map of the same splitting degree.

Arrows over crossings Choose link diagrams $D$ and $D^{\prime}$ that differ only in the direction of arrows decorating the crossings. Then $\mathcal{I}\left(D^{\prime}\right)$ is the cube $\mathcal{I}(D)$ with some edges scaled by $X$ or $Y$, due to (3). These coefficients define a cochain $\lambda \in C^{1}\left(I^{n} ; \mathbb{k}^{*}\right)$, and if $\epsilon$ is a sign assignment for $\mathcal{I}(D)$, so is $\epsilon \lambda^{-1}$ for $\mathcal{I}\left(D^{\prime}\right)$. One can easily see that

$$
\mathcal{I}^{\epsilon}(D)=\mathcal{I}^{\epsilon \lambda^{-1}}\left(D^{\prime}\right) .
$$

Orderings on crossings and circles A change in enumeration of crossings permutes only the direct summands in (9). On the other hand, each component of the isomorphism of cubes that reorders circles in resolutions is a composition of twists. Hence it is homogeneous, and we again use Lemma 4.4 to deduce that all components have the same splitting degree.

Corollary 4.5 The isomorphism class of the graded generalized Khovanov bracket $\llbracket D \rrbracket$ depends only on the link diagram $D$.

We shall now proceed to Reidemeister moves and show that the chain homotopy equivalences defined in [6, Section 7] are homogeneous. We shall recall how they are defined, but for clarity all cobordisms are drawn without arrows orienting their critical points. The convention to keep in mind is that deaths are negative, whereas arrows orienting merges and splits point towards right or front.

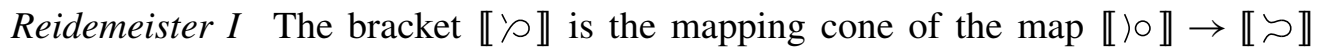
induced by edges in the cube $\mathcal{I}(\supset)$ associated to the distinguished crossing. The chain homotopy equivalences between complexes $\llbracket \curvearrowright \rrbracket$ and $\llbracket \supset \rrbracket$ are induced by 
morphisms of cubes $f: \mathcal{I}(\supset) \rightleftarrows \mathcal{I}() \circ): g$ as shown in the diagram below:

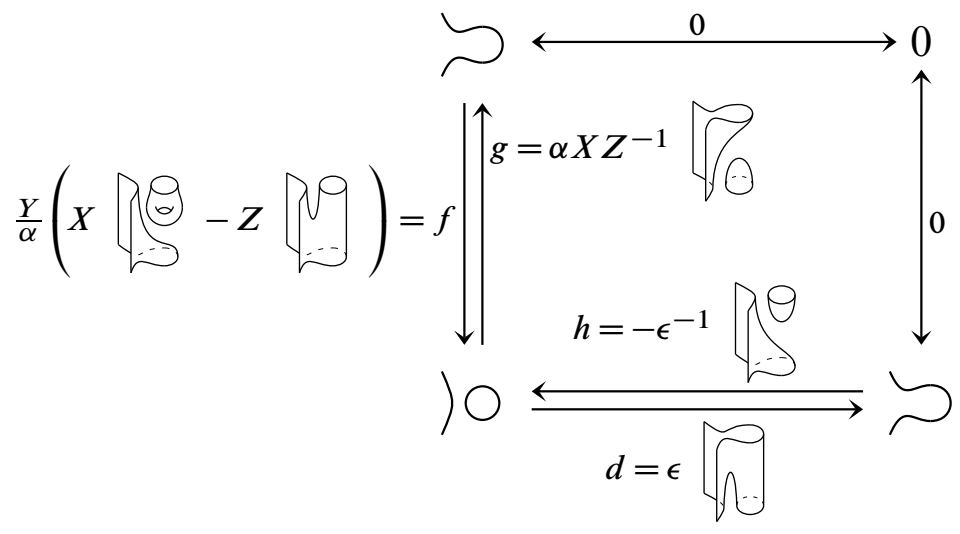

Here, $\epsilon$ comes from the sign assignment used to build $\llbracket \supset \rrbracket$, and $\alpha \in \mathbb{k}$ is chosen for each component of $f$ and $g$ separately, to make them commute with other edge morphisms in the cubes (see [6, proof of Theorem 7.1] for more details). It follows directly from Lemma 4.4 that $g$ induces a homogeneous chain map, and for $f$ we have to check that the two cobordisms have the same degree. Indeed,

$$
\begin{aligned}
& \operatorname{sdeg}\left(Z\left[\begin{array}{r}
\mathcal{P} \\
\ldots
\end{array}\right)=\left[\begin{array}{r}
0 \\
-1
\end{array}\right]+\left[\begin{array}{r}
0 \\
-2
\end{array}\right]=\left[\begin{array}{r}
0 \\
-3
\end{array}\right],\right. \\
& \operatorname{sdeg}(X)\left(\begin{array}{l}
1 \\
0
\end{array}\right]+\left[\begin{array}{l}
0 \\
0
\end{array}\right]+\left[\begin{array}{l}
-1 \\
-3
\end{array}\right]+\left[\begin{array}{l}
0 \\
0
\end{array}\right]=\left[\begin{array}{r}
0 \\
-3
\end{array}\right] \text {, }
\end{aligned}
$$

after forgetting the circles not shown in the diagrams, and placing the circle drawn in full as the first one.

Reidemeister II Homotopy equivalences for the second move are shown in Figure 2. Again, we look on $\llbracket \curvearrowright ' \rrbracket$ as the total complex of $\llbracket) \sim \rrbracket \rightarrow \llbracket) \circ \rrbracket \oplus \llbracket \approx \rrbracket \rightarrow \llbracket \precsim(\rrbracket$. Although it looks more challenging, the way the morphisms $f$ and $g$ are defined makes the proof very easy. Indeed, the morphisms between $\asymp$ and )o ( are compositions of edge morphisms and homotopies: $f_{01}=h_{* 1} d_{1 *}$, and $g_{01}=d_{* 0} h_{0 *}$. Taking into account the degree shifts, the differentials and homotopies preserve sdeg, which implies both $f_{01}$ and $g_{01}$ have the same degree as the morphisms between $\asymp$ and $\approx$ (which is homogeneous, but possibly of a nontrivial degree).

Reidemeister III Invariance under the last move followed from a strictly algebraic

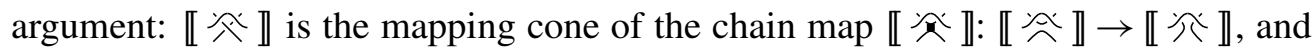

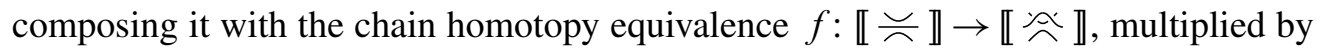



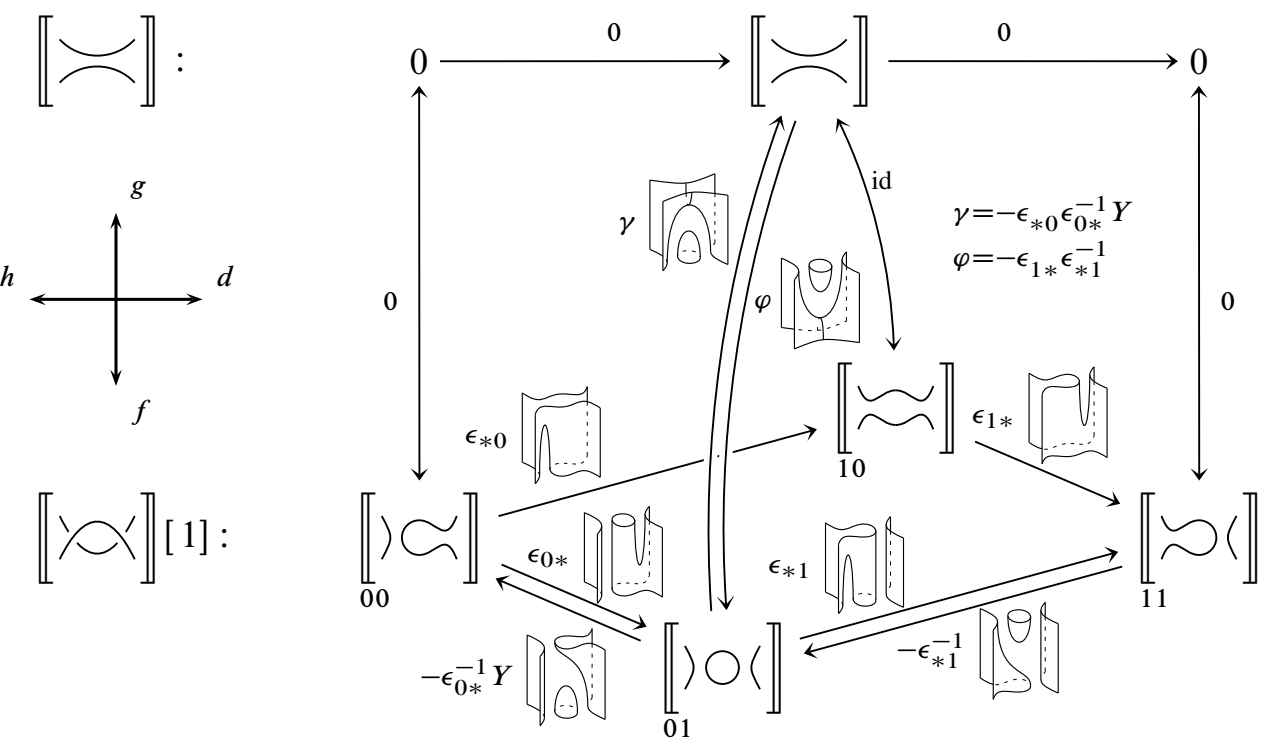

Figure 2: Chain homotopy equivalences for the second Reidemeister move

a certain monomial $X^{a} Z^{b}$ to make it graded, does not change the homotopy type

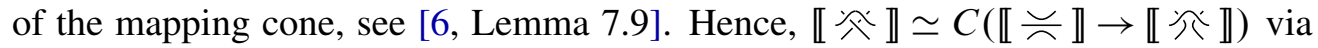
degree-preserving chain homotopy equivalences, and similarly for $\llbracket \mathbb{~} \rrbracket$.

This ends the proof of Theorem 4.1 in the view of Lemma 4.3.

\section{Applications}

\subsection{Reduction of parameters}

Let $\mathbb{k}_{0} \subset \mathbb{k}$ be the subring consisting of the elements in splitting degree zero. It is generated by $X Y$, and as such it isomorphic to $\mathbb{Z}_{\pi}:=\mathbb{Z}[\pi] /\left(\pi^{2}-1\right)$. On the other hand, there is a ring epimorphism $\mathbb{k} \rightarrow \mathbb{Z}_{\pi}$ sending both $X$ and $Z$ to 1 , and $Y$ to $\pi$. In particular, we can construct $\mathbb{Z}_{\pi} \mathbf{C h C o b}$, see Remark 2.5.

Lemma 5.1 The pair of $\mathbb{k}_{0}$-linear functors $I: \mathbb{Z}_{\pi} \mathbf{C h C o b} \leftrightarrows \mathbb{k}_{\mathbf{C h C o b}}: P$,

$$
\begin{aligned}
I(\Sigma) & :=\Sigma\{0,0\}, & P(\Sigma\{a, b\}) & :=\Sigma, \\
I\left(\pi^{k} W\right) & :=X^{a+k} Y^{k} Z^{b} W, & P\left(X^{p} Y^{q} Z^{r} W\right) & :=\pi^{q} W,
\end{aligned}
$$

for $\operatorname{sdeg} W=\left[\begin{array}{l}a \\ b\end{array}\right]$, is an equivalence of categories. 
Proof Clearly $P I=\mathrm{id}$, and the natural isomorphism id $\cong I P$ is given by the family of scaled cobordisms

$$
\Sigma\{a, b\} \stackrel{X^{a} Z^{b}(\Sigma \times I)}{\longrightarrow} \Sigma .
$$

Let $K h_{\pi}(D)$ stand for the generalized Khovanov complex built in $\operatorname{Mat}\left(\mathbb{Z}_{\pi} \mathbf{C h C o b}\right)$. Clearly, $\mathcal{F} K h\left(D ; \mathbb{Z}_{\pi}\right) \cong \mathcal{F}_{\pi} K h_{\pi}(D)$, where $\mathcal{F}_{\pi}: \mathbb{Z}_{\pi} \mathbf{C h C o b} \rightarrow \operatorname{Mod}_{\mathbb{Z}_{\pi}}$ is defined similarly to $\mathcal{F} .^{5}$

Corollary 5.2 The generalized Khovanov complexes $K h(D)$ and $K h_{\pi}(D)$ are equivalent link invariants: $K h(D) \simeq K h\left(D^{\prime}\right)$ for link diagrams $D$ and $D^{\prime}$ if and only if $K h_{\pi}(D) \simeq K h_{\pi}\left(D^{\prime}\right)$.

There is a similar equivalence between $\operatorname{Mod}_{\mathbb{k}, 0}$ and $\operatorname{Mod}_{\mathbb{Z}_{\pi}}$. Extracting the degree 0 component $M_{0}$ of a $\mathbb{k}$-module $M$ results in a functor $r: \operatorname{Mod}_{\mathbb{k}, 0} \rightarrow \operatorname{Mod}_{\mathbb{Z}_{\pi}}$. Conversely, given a $\mathbb{Z}_{\pi}$-module $N$ one creates a $\mathbb{k}$-module $i(N):=\bigoplus_{(a, b) \in \mathbb{Z}_{2} \times \mathbb{Z}} N$, where $(X Y) \cdot n=\pi n$, while $X$ and $Z$ permute the copies of $N$ in $i(N)$.

Lemma 5.3 The pair of functors $i: \operatorname{Mod}_{\mathbb{Z}_{\pi}} \leftrightarrows \operatorname{Mod}_{\mathbb{k}, 0}: r$ is an equivalence of categories.

Proof Straightforward.

The above equivalences intertwine the two TQFT functors $\mathcal{F}: \mathbb{k} \mathbf{C h C o b}_{0} \rightarrow \mathbf{M o d}_{\mathbb{k}, 0}$ and $\mathcal{F}_{\pi}: \mathbb{Z}_{\pi} \mathbf{C h C o b} \rightarrow \operatorname{Mod}_{\mathbb{Z}_{\pi}}$, resulting in a direct connection between $\mathcal{H}(D)$ and $\mathcal{H}\left(D ; \mathbb{Z}_{\pi}\right)$.

Theorem 5.4 (the reduction of parameters) The generalized Khovanov complex $\mathcal{F} K h(D)$, regarded as a complex of $\mathbb{Z}_{\pi}$-modules, decomposes into a direct sum of subcomplexes

$$
\mathcal{F} K h(D) \cong \bigoplus_{(a, b) \in \mathbb{Z}_{2} \times \mathbb{Z}} \mathcal{F} K h(D)_{a, b},
$$

each isomorphic to $\mathcal{F} K h\left(D ; \mathbb{Z}_{\pi}\right) \cong \mathcal{F}_{\pi} K h_{\pi}(D)$. The action of $\mathbb{k}$ is given by isomorphisms

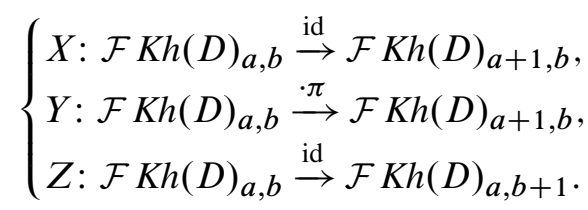

${ }^{5}$ Think of $\mathcal{F}_{\pi}$ as the tensor product $\mathcal{F} \otimes \mathbb{Z}_{\pi}$ over $\mathbb{k}$. 
Proof The decomposition follows from Theorem 4.1, so it remains to compute the degree zero subcomplex. First, $r(M)$ is naturally isomorphic to $M \otimes \mathbb{Z}_{\pi}$ via $m \mapsto m \otimes 1$. Indeed, this map is linear over $\mathbb{Z}_{\pi}$, and its inverse sends $m \otimes 1$, with $\operatorname{sdeg}(m)=\left[\begin{array}{l}a \\ b\end{array}\right]$, into $X^{a} Z^{b} m$. Hence, $\mathcal{F} K h(D)_{0,0}$ is naturally isomorphic to $\mathcal{F} K h(D) \otimes \mathbb{Z}_{\pi}=\mathcal{F} K h\left(D ; \mathbb{Z}_{\pi}\right)$.

Given a graded ring automorphism $\varphi \in \mathrm{Aut}_{0}(\mathbb{k})$ we can replace the chronological parameters $X, Y$ and $Z$ with their images under $\varphi$, resulting in a graded category $\mathbb{k}_{\varphi} \mathbf{C h C o b}_{0}$ and a chronological TQFT $\mathcal{F}_{\varphi}: \mathbb{k}_{\varphi} \mathbf{C h C o b}_{0} \rightarrow \mathbf{M o d}_{\mathbb{k}, 0}$. As before, given a link diagram $D$ we can construct the generalized Khovanov complex $K h_{\varphi}(D)$ in $\operatorname{Mat}\left(\mathbb{k}_{\varphi} \mathbf{C h C o b}\right)$. In view of Corollary 5.2, the complexes $\mathcal{F} K h(D)$ and $\mathcal{F}_{\varphi} K h_{\varphi}(D)$ are equivalent link invariants if $\varphi(X Y)=X Y$ (ie if $\left.\varphi\right|_{\mathbb{k}_{0}}=\mathrm{id}$ ). We shall now show they are in fact isomorphic.

Proposition 5.5 Assume $\varphi(X Y)=X Y$. Then the complexes of $\mathbb{k}$-modules $\mathcal{F} K h(D)$ and $\mathcal{F}_{\varphi} K h_{\varphi}(D)$ are isomorphic for any link diagram $D$.

Proof Decompose the complexes as in Theorem 5.4. $\mathcal{F} K h(D)_{0,0}$ and $\mathcal{F}_{\varphi} K h_{\varphi}(D)_{0,0}$ are complexes of free $\mathbb{Z}_{\pi}$-modules, and $\varphi$ induces an isomorphism between them. Indeed, $\pi$ acts on both complexes as multiplication by $X Y=\varphi(X Y)$. Thence, it is enough to extend the equality in a $\mathbb{k}$-linear way. Explicitly,

$$
\mathcal{F} K h(D) \rightarrow \mathcal{F}_{\varphi} K h_{\varphi}(D), \quad u \mapsto\left(\frac{\varphi(X)}{X}\right)^{a}\left(\frac{\varphi(Z)}{Z}\right)^{b} u
$$

for a generator $u=v_{i_{1}} \otimes \cdots \otimes v_{i_{k}}$ in degree $\operatorname{sdeg}(u)=\left[\begin{array}{l}a \\ b\end{array}\right] .{ }^{6}$

Denote by $\mathbb{k}_{\varphi}$ the ring $\mathbb{k}$ with a module structure twisted by $\varphi$, ie $k \cdot x:=\varphi(k) x$. Every $\mathbb{k}$-module structure on $\mathbb{Z}$ can be obtained by taking a tensor product $\mathbb{k}_{\varphi} \otimes \mathbb{Z}_{e v}$ or $\mathbb{k}_{\varphi} \otimes \mathbb{Z}_{\text {odd }}$ for an automorphism $\varphi$ fixing $X Y$. For instance, if $\varphi(X)=-X$ and likewise for $Y$ and $Z$, then each parameter acts on $\mathbb{Z}^{\prime}:=\mathbb{k}_{\varphi} \otimes \mathbb{Z}_{e v}$ as -1 .

Corollary 5.6 Given a $\mathbb{k}$-module structure on $\mathbb{Z}$, the homology $\mathcal{H}(L ; \mathbb{Z})$ is either the even Khovanov homology, if $X Y$ acts on $\mathbb{Z}$ as identity, or the odd Khovanov homology otherwise.

Remark 5.7 The even and odd Khovanov homology are not equivalent. Hence, the condition on $\varphi$ in Proposition 5.5 is necessary.

\footnotetext{
${ }^{6}$ Here, $\operatorname{sdeg}(u)$ is the degree of $u$ as an element of the graded complex $\mathcal{F} K h$, and it can be different from when $u$ is regarded as an element of $A^{\otimes k}$.
} 
Remark 5.8 Theorem 5.4 holds for any chronological Frobenius system $(R, A)$ with $R$ supported in weight 0 . In particular, we can take the algebra of dotted chronological cobordisms $\left(R_{\bullet}, A_{\bullet}\right)$, defined by one of the authors in [6, Section 11], in which $R_{\bullet}$ is generated over $\mathbb{k}$ by $h$ and $t$ of degrees $\operatorname{deg} h=(-1,-1)$ and $\operatorname{deg} t=(-2,-2)$.

\subsection{Duality}

Choose a link diagram $D$. The Khovanov homology of the mirror image $D^{!}$is dual to the one of $D$, see Khovanov [2]. Namely, there is an isomorphism of complexes

$$
\mathcal{F}_{\text {ev }} K h\left(D^{!}\right) \cong \operatorname{Hom}\left(\mathcal{F}_{e v} K h(D), \mathbb{Z}\right),
$$

which follows from the following two observations.

(1) Resolutions of crossings in $D^{\text {! }}$ are those of $D$, but with type 0 and type 1 interchanged, as illustrated below:

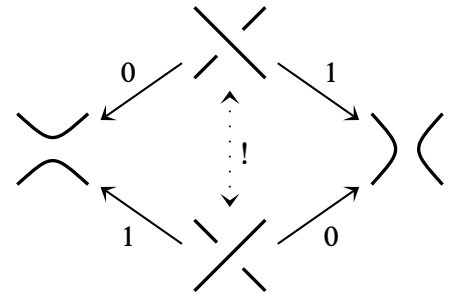

In particular, the cube $\mathcal{I}\left(D^{!}\right)$looks like $\mathcal{I}(D)$, but with all arrows reversed.

(2) The Khovanov algebra $A$ is self-dual: $A^{*} \cong A$ via $v_{ \pm}^{*} \mapsto v_{\mp}$.

A similar phenomenon occurs in the generalized case [6, Proposition 8.3] with a few differences. The algebra $A$ over $\mathbb{k}$ is not strictly self-dual: $A^{*} \cong \bar{A}$ as algebras over $\overline{\mathbb{k}}$, where we exchange the roles of $X$ and $Y .{ }^{7}$ For instance,

$$
\begin{aligned}
\Delta^{*}\left(v_{+}^{*} \otimes v_{-}^{*}\right) & =Y Z v_{+}^{*}, \\
\mu^{*}\left(v_{-}^{*}\right) & =v_{+}^{*} \otimes v_{-}^{*}+X Z v_{-}^{*} \otimes v_{+}^{*} .
\end{aligned}
$$

Likewise, the duality between cubes $\mathcal{I}\left(D^{!}\right)$and $\mathcal{I}(D)$ is realized by an operation on chronological cobordisms (_)*: $\mathbb{k} \mathbf{C h C o b} \rightarrow \overline{\mathbb{k}} \mathbf{C h C o b}$ that "reverses" the chronology, ie $(W, \tau)^{*}:=\left(W, \tau^{*}\right)$ with $\tau^{*}(t):=1-\tau(t)$. Reversing a cobordism permutes its degree components, $\operatorname{deg} W^{*}=(b, a)$ if $\operatorname{deg} W=(a, b)$, but it also intertwines the two disjoint unions: $\left(W \downarrow \downarrow W^{\prime}\right)^{*}=W^{*} \downarrow W^{\prime *}$. This explains why the roles of $X$ and $Y$ are exchanged, but the role of $Z$ is preserved.

${ }^{7}$ In other words, $\overline{\mathbb{k}}=\mathbb{k}_{\varphi}$, where $\varphi(X)=Y, \varphi(Y)=X$ and $\varphi(Z)=Z$. 
When reversing the chronology of a cobordism one must also take care of orientations of critical points. Indeed, an orientation of a critical point $p \in W$ induces an orientation of the stable part of $T_{p} W^{*}$. We choose for the unstable part the complementary orientation with respect to the outward orientation of the cobordism $W$. Diagrammatically, color each region in the complement of $W$ black or white, so that the unbounded region is white and regions with same colors do not meet; then for a saddle point $p$ rotate the framing arrow in $W^{*}$ clockwise if the region below $p \in W$ is white, and anticlockwise otherwise:
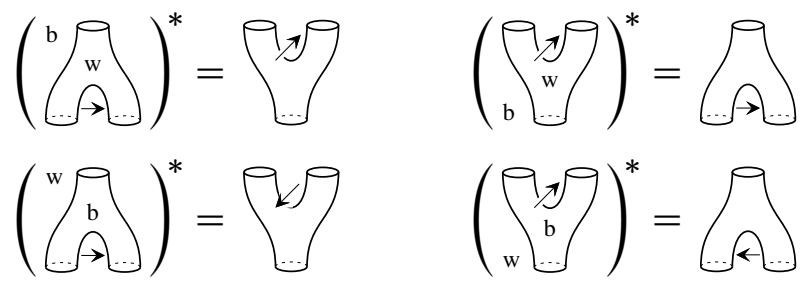

Since we want the duality functor to be coherent with (3) there is no choice left for births and deaths:

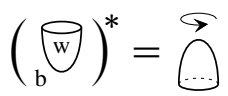

$$
\begin{aligned}
& (\stackrel{\mathrm{b}}{\mathrm{w}})^{*}=\emptyset \\
& (\stackrel{\mathrm{b}}{\mathrm{w}})^{*}=Y \Theta \\
& (\mathrm{b}))^{*}=\stackrel{?}{\mathrm{C}} \\
& \left({ }^{\mathrm{w}} \stackrel{\mathrm{b}}{\mathrm{b}}\right)^{*}=\theta \\
& (\stackrel{\mathrm{w}}{\mathrm{b}})^{*}=Y \vartheta
\end{aligned}
$$

We showed in [6, Section 8] that $\mathcal{I}^{\epsilon}\left(D^{!}\right)$, regarded as an object in $\mathbf{K o m}(\overline{\mathbb{k}} \mathbf{C h C o b})$, is the image of $\mathcal{I}^{\epsilon}(D)$ under the above operation, so $\mathcal{F}_{\overline{\mathbb{k}}} K h_{\overline{\mathbb{k}}}\left(D^{!}\right) \cong \mathcal{F} K h(D)^{*}$. The results of the previous section allow us to switch $\overline{\mathbb{k}}$ back to $\mathbb{k}$.

Theorem 5.9 (duality for generalized Khovanov homology) Given a link diagram $D$ and its mirror image $D^{!}$there is an isomorphism of complexes

$$
\mathcal{F} K h\left(D^{!}\right) \cong \mathcal{F} K h(D)^{*},
$$

where $\left(C^{*}\right)^{i}:=\operatorname{Hom}\left(C^{-i}, \mathbb{k}\right)$ for a chain complex $C$. In particular, the odd Khovanov homology $\mathcal{H}_{\text {odd }}(L)$ of a link $L$ is dual to $\mathcal{H}_{\text {odd }}\left(L^{!}\right)$, and similarly for $\mathcal{H}_{\pi}(L)$ and $\mathcal{H}_{\pi}\left(L^{!}\right)$.

Proof Proposition 5.5 and the discussion above give a sequence of isomorphisms

$$
\mathcal{F} K h\left(D^{!}\right) \cong \mathcal{F}_{\overline{\mathbb{k}}} K h_{\overline{\mathbb{K}}}\left(D^{!}\right) \cong \mathcal{F} K h(D)^{*}
$$


The cases of $\mathcal{H}_{o d d}$ and $\mathcal{H}_{\pi}$ follow from the existence of an isomorphism

$$
\operatorname{Hom}_{\mathbb{k}}(F, \mathbb{k}) \otimes R \cong R^{\mathrm{rk} F} \cong \operatorname{End}_{R}(R)^{\mathrm{rk} F} \cong \operatorname{Hom}_{R}(F \otimes R, R)
$$

for any free module $F$ of finite rank and $\mathbb{k}$-algebra $R$.

The duality isomorphism (43) is given explicitly as

$$
\mathcal{F}\left(D_{\zeta}^{!}\right) \rightarrow \mathcal{F}\left(D_{\bar{\zeta}}\right)^{*}, \quad u \mapsto(X Y)^{a} u^{*}
$$

where $u=v_{i_{1}} \otimes \cdots \otimes v_{i_{k}}$ has degree $\operatorname{sdeg} u=\left[\begin{array}{l}a \\ b\end{array}\right]$. For the other version of Khovanov homology simply replace $X Y$ with either $\pi$, for the unified homology, or $(-1)$ for the odd one. Note the role of the splitting degree: although it does not descend directly to $\mathcal{H}_{\text {odd }}(L)$ or $\mathcal{H}_{\pi}(L)$, it controls the duality isomorphism.

\section{References}

[1] D Bar-Natan, Khovanov's homology for tangles and cobordisms, Geom. Topol. 9 (2005) 1443-1499 MR2174270

[2] M Khovanov, A categorification of the Jones polynomial, Duke Math. J. 101 (2000) 359-426 MR1740682

[3] M Khovanov, An invariant of tangle cobordisms, Trans. Amer. Math. Soc. 358 (2006) 315-327 MR2171235

[4] PS Ozsváth, J Rasmussen, Z Szabó, Odd Khovanov homology, Algebr. Geom. Topol. 13 (2013) 1465-1488 MR3071132

[5] K K Putyra, Cobordisms with chronologies and a generalization of the Khovanov complex, master's thesis, Jagiellonian University, Krakow (2008) arXiv:1004.0889

[6] K K Putyra, A 2-category of chronological cobordisms and odd Khovanov homology, from: "Knots in Poland III, Part 3", (J H Przytycki, P Traczyk, editors), Banach Center Publ. 103, Polish Acad. Sci. Inst. Math., Warsaw (2014) 291-355 MR3363817

[7] A N Shumakovitch, Patterns in odd Khovanov homology, J. Knot Theory Ramifications 20 (2011) 203-222 MR2777025

Institute for Theoretical Studies, ETH Zürich

Clausiusstrasse 47, CH-8092 Zurich, Switzerland

Theoretical Computer Science Department, Faculty of Mathematics and Computer Science, Jagiellonian University

ul. Lojasiewicza 6, 30-348 Kraków, Poland

krzysztof.putyra@eth-its.ethz.ch, w.lubawski@gmail.com

Received: 10 February 2015 Revised: 18 September 2015 